\title{
Palmier à huile : le management environnemental des plantations
}

\author{
Le cheminement de PT. Smart
}

Jean-Pierre CALIMAN

Directeur de Smart Research Institute

(SMARTRI) ;

Chercheur Cirad,

Détaché à PT. Smart

Po Box 1340

28000 Pekanbaru - Riau

Indonésie

<j-p-caliman@smart-tbk.com>

\begin{abstract}
PT. Smart, an Indonesian oil palm plantations company, technically manages all plantings of Golden Agri-Resources (GAR). Recently challenged by several environmental NCOs, accusing the society of violating the durability, despite its accession to the panel for the production of sustainable palm oil (RSPO), the company has re-affirmed its commitment, strengthening its governance with the goal of becoming not only a leader in its field of production, but also in terms of environmental and social sustainability, implementing coordination activities and exchanges to find workable solutions to problems. Recognizing that some mistakes were made, the company insists, however, that many economic initiatives, social and environmental development marked its action since the early 1980s according to the advance of scientific knowledge, and the existence of operational tools to implement them. What the company calls "the road towards sustainability".
\end{abstract}

Key words: sustainability, oil palm, plantation

ne pas ne prendre suffisamment en compte les aspects de durabilité. Ce mouvement, rapidement intensifié au cours des 10 dernières années, s'organisait autour des thèmes du changement climatique (comprenant l'émission de gaz à effet de serre liés à la déforestation), de la perte de la biodiversité, des risques de pollution et dégradation du milieu, mais aussi du respect des droits sociaux relatifs aux populations locales et aux travailleurs. Pour le palmier à huile, I'aspect nutritionnel ressurgissait également, en écho aux campagnes conduites au début des années 1980 par le lobby du soja, alors effrayé par les capacités de productivité du palmier à huile.

Ces campagnes intervenaient également à un moment où les premières sociétés de plantations de palmiers à huile mettaient en route progressivement un processus de certification de durabilité des unités de production, suivant les principes et critères $(P \& C)$, les indicateurs et guidelines, définis en 2007 par la table ronde pour la production d'huile de palme durable (www.rspo.org). La stratégie opérationnelle de la plupart des sociétés ayant adhéré à cette initiative était de construire une feuille de route pour la certification de l'ensemble des unités de production dans un espace de temps compatible avec les P\&C établis dans RSPO. Cette feuille de route comprenait généralement une première phase d'évaluation de l'écart entre les pratiques de production et les $P \& C$ de durabilité, une deuxième phase de mise à niveau de ces pratiques, lorsque nécessaire, et une phase de certification proprement dite. La constitution d'une structure dédiée et la formation du personnel capable de gérer les trois phases constituaient un challenge à relever, mais indispensable pour le succès de ces plans. Cependant, s'appuyant sur le principe de continuous improvement, les sociétés ayant adhéré à RSPO étaient relativement confiantes sur leur capacité à être progressivement en conformité avec les P\&C, d'autant plus qu'elles estimaient qu'un nombre significatif de $P \& C$ étaient déjà prescrits dans leurs Standard Operating Procedures (SOP) et donc déjà en application dans leurs unités opérationnelles.

Pour citer cet article : Caliman JP. Palmier à huile : le management environnemental des plantations. Le cheminement de PT. Smart. OCL 2011 ; 18(3) : 123-31. doi : 10.1684/ocl.2011.0380 
Cette stratégie était également celle adoptée par PT. Smart depuis son adhésion à RSPO en 2005. La société était prête à relever le défit, forte de sa capacité démontrée d'adapter ses techniques de production aux standards de productivité démontrés. Depuis les débuts de son développement, il y a une trentaine d'années, la société avait mis en œuvre progressivement, en fonction des connaissances du moment et de la disponibilité d'outils opérationnels, les pratiques ou, lorsque jugé nécessaire, des recherches liées directement ou indirectement aux 3 aspects relatifs à la durabilité.

Cependant, il s'est avéré que le rythme pris par un certain nombre de sociétés, et la notion de "continuous improvement ", n'avait pas la même valeur pour certaines ONG souhaitant des changements ou adaptations des pratiques plus rapides.

L'objectif de cet article est de montrer, sans chercher à être exhaustif, comment PT. Smart a progressivement, depuis le début de son développement il y a près de trente ans, mis en place des pratiques et des études constituant la route vers la production durable d'huile de palme; comment cette société, reconnaissant par ailleurs certaines erreurs, a répondu aux attaques des ONG environnementales en réaffirmant ses engagements, et en renforçant sa gouvernance, avec l'objectif de devenir non seulement I'un des leaders dans son domaine de production, mais aussi concernant les autres piliers de la durabilité, en mettant en œuvre des actions de coordination et d'échanges afin de trouver des solutions opérationnelles aux problèmes posés.

\section{Le développement du palmier à huile dans le monde et en Indonésie}

Le palmier à huile : $7 \%$

des surfaces agricoles

en oléagineux assurent $39 \%$

de la production mondiale en

huile végétale

Avec environ 13 millions d'hectares, le palmier à huile couvre $7 \%$ des surfaces agricoles consacrées à la production d'huile végétale, soit de loin la plus petite portion comparée au soja (61\%), colza (18\%) et tournesol (14\%) (figure $1 A$ ). Cependant sa production (46,7 millions de tonnes en 2010 ) représente $39 \%$ de la production mondiale $d$ 'huile végétale (120 millions de tonnes), devançant le soja (32 \%) et loin devant le colza (19 \%) et le tournesol (10\%) (figure 1B) (Oil World, 2010).

$C^{\prime}$ est dire le potentiel de cette culture, dont il faut de plus garder en mémoire qu'une part importante des surfaces récemment développées n'a encore pas atteint le niveau de production attendu. Par ailleurs, des augmentations significatives de production peuvent être espérées par une meilleure gestion de certaines surfaces déjà cultivées (utilisation de matériel végétal sélectionné, gestion optimale des intrants, mise en place de services de vulgarisation, etc.), notamment chez nombre de petits planteurs indépendants n'ayant souvent que peu bénéficié des progrès de la recherche du fait de services de vulgarisation souvent inexistants. Rappelons qu'environ $60 \%$ des surfaces cultivées en palmiers à huile dans le monde sont gérées par des petits planteurs villageois.

Ces chiffres donnent aussi une indication des besoins en surfaces agricoles nouvelles qui devraient être consacrées à la production $d$ 'huile végétale pour subvenir aux besoins croissant d'une population en développement: toute augmentation de la demande en huile végétale de 10 millions de tonnes (soit $8 \%$ de la production actuelle) deman-

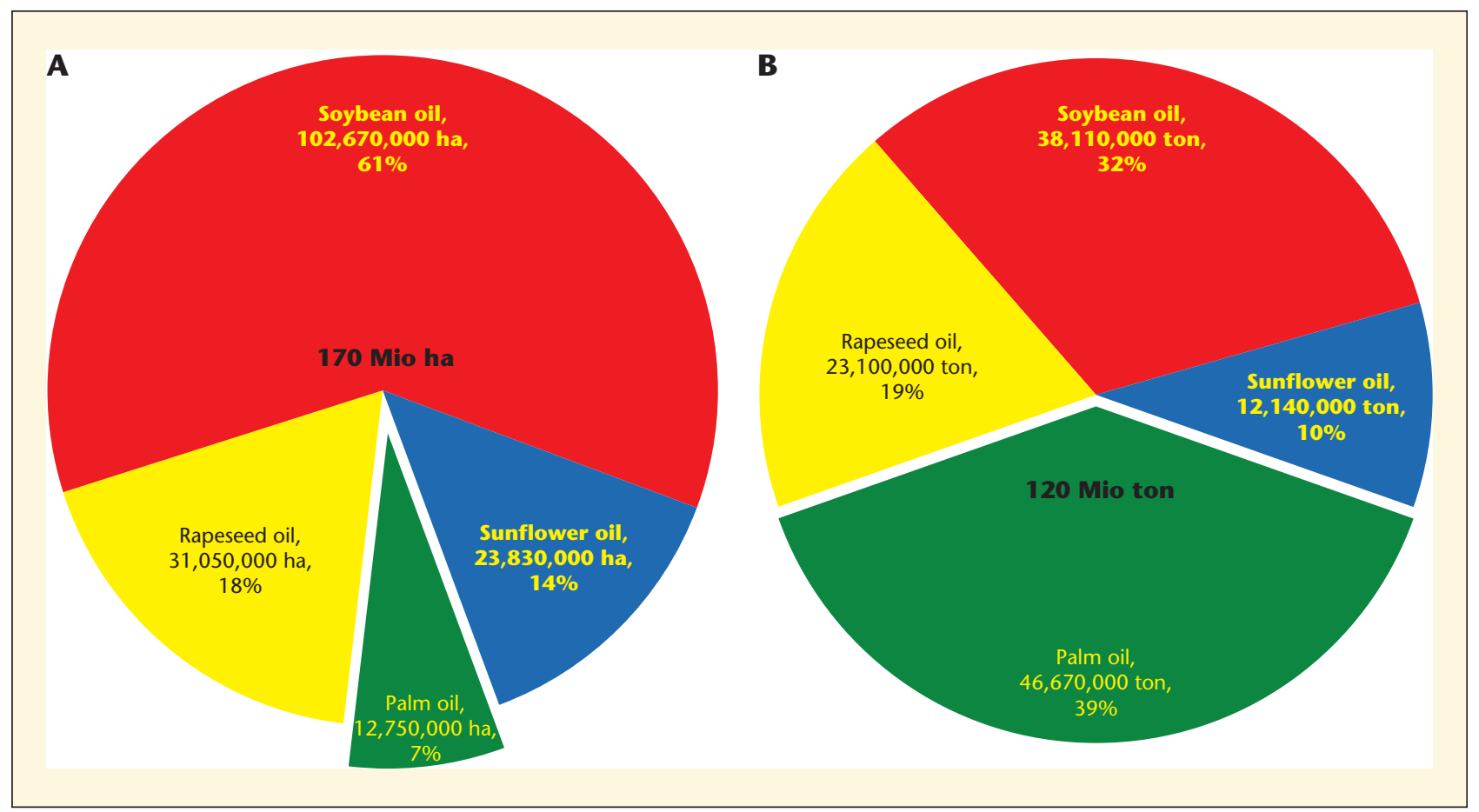

Figure 1. A) Surfaces agricoles consacrées aux principales cultures oléagineuses (Oil World, 2010. B) Production des principales huiles végétales (Oil World, 2010). 

avoisinant 2,5 millions d'hectares pour le palmier à huile, 15 millions d'hectares pour le colza, 20 millions d'hectares pour le tournesol et plus de 25 millions d'hectares pour le soja (figure 2) suivant les cultures choisies pour cette production.

\section{La culture du palmier à huile : une situation géographique sensible et un développement récent sous les feux des projecteurs}

Cette haute productivité aurait dû placer le palmier à huile dans une situation idéale si ce n'était le fait qu'un développement important des surfaces s'est effectué au cours des deux dernières décennies, dont une partie significative sur couvert forestier, et exclusivement en zone tropicale. L'Indonésie, par exem7,5 millions d'hectares au cours des 20 dernières années. Une part significative a derait un accroissement des surfaces ple, a développé plus de $80 \%$ de ses

sans nul doute été développée sur forêt, même si cela ne représente qu'une portion des 24 millions $d$ 'hectares déforestés sur cette période notamment pour I'utilisation du bois (FAO, 2011). En contraste, les cultures oléagineuses des pays occidentaux, telles que le colza, tournesol et une partie du soja, sont cultivées sur les surfaces déboisées il y a souvent plusieurs siècles.

Cette période correspond également à la prise de conscience de l'impact environnemental d'un développement fondé sur les schémas successifs utilisés au cours de la deuxième moitié du $X X^{\mathrm{e}}$ siècle.

Avec comme objectif affiché une production doublée en 2020, soit 40 millions de tonnes d'huile de palme, tout en suivant ses engagements de réduction de la déforestation et d'émission de gaz à effet de serre de $26 \%$ sur la période, I'Indonésie, premier producteur mondial est face à un véritable challenge dont la solution consistera à orienter ses futurs développements de nouvelles planta-

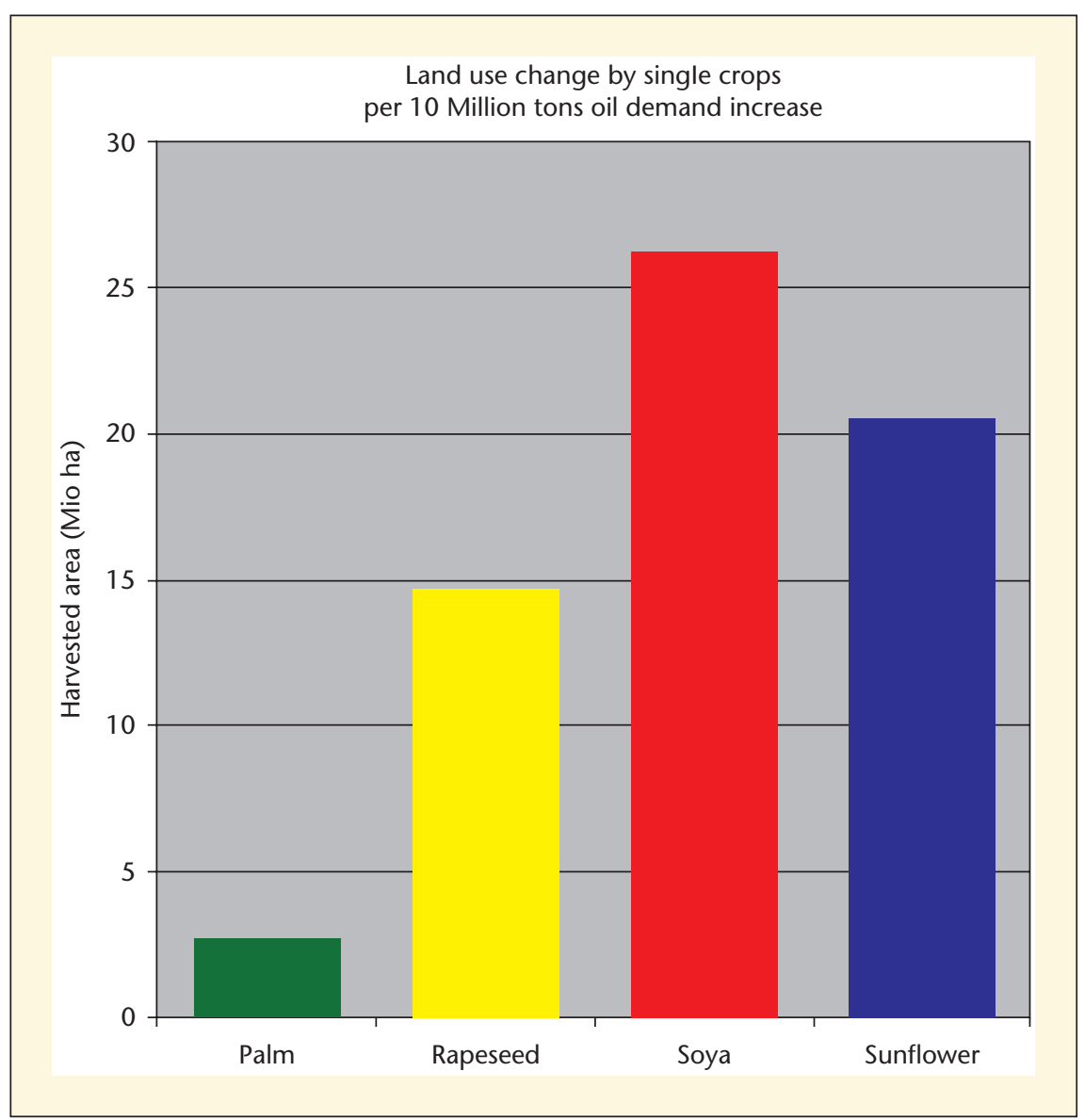

Figure 2. Surfaces agricoles nécessaire à la production de 10 millions de tonnes d'huile: végétales suivant les cultures (Oil World, 2010). tions vers des zones dégradées et d'autre part à augmenter la productivité des surfaces déjà plantées. Ces deux possibilités paraissent réalisables, étant donné les surfaces dégradées disponibles, et considérant le potentiel de production actuel du pays, dont la moyenne de la production nationale $(3,52 \mathrm{t} / \mathrm{ha})$ enregistrée en 2009 (IPOC, 2009) n'atteignait que $2 / 3$ de niveau moyen de production d'une société telle que PT. SMART (GAR, 2009).

\section{Golden agri resources (GAR) - PT. Smart}

Avec plus de 442000 ha plantés à fin 2010, dont $20 \%$ de plantations appartenant à des petits planteurs dont le développement a été associé a celui de la compagnie (voir plus loin), GAR est I'un des leaders de l'industrie du palmier à huile (encadré 1). Sa structure totalement intégrée, allant d'un centre de Recherches \& Développement, de la production de semences à haut potentiel de rendement, couvrant les plantations de palmiers, les usines d'extraction d'huile, les raffineries, jusqu'à la vente de produits transformés et finis sous marques propres, permet à la société une performance reconnue parmi les meilleures de l'industrie, aussi bien sur les surfaces qui lui appartiennent en propre que sur les terrains appartenant aux petits planteurs dont la société assure l'encadrement, après avoir contribué au développement. En 2009, la production moyenne s'est établie à 5,36 tonnes d'huile à l'hectare, soit plus de $52 \%$ audessus de la moyenne nationale pour I'Indonésie (3,5 tonnes/ha).

Société emblématique de la culture du palmier à huile en Indonésie, depuis l'expansion de cette culture dans les années 1980, la compagnie s'est développée à un rythme rapide, reflétant le dynamisme de ses dirigeants et la volonté de l'Indonésie de s'appuyer sur l'agriculture afin de réduire le niveau de pauvreté du pays. Par ailleurs, les performances de production enregistrées sont liées au niveau de technicité acquis par l'ensemble des employés au cours de programmes de formation adaptés et la mise en place de pratiques de production appropriées (matériel végétal performant, efficience des intrants, lutte intégrée contre les ravageurs, etc.) toujours renouvelées en fonction de l'avancée des connaissances. 


\section{Golden Agri-Resources : descriptif de la compagnie}

Golden Agri-Resources (www.goldenagri.com.sg) :

- Surfaces cultivées en palmier à huile : 442000 ha

- Capacité d'usinage : 9,27 millions tonnes de régimes par an ; 36 usines d'extraction d'huile de palme et de palmiste.

- Capacité des raffineries : 1,38 million de tonnes par an.

- Revenu : 3,5 milliards de dollars US

- Production moyenne : 5,91 tonnes d'huile (huile de palme + huile de palmiste) par hectare récoltés en 2009, (plus de $50 \%$ au-dessus de la moyenne nationale en Indonésie). Les petits planteurs associés à la compagnie enregistrant une production parfois supérieure.

- En 2010, l'entreprise a consacrée $\mathbf{3 , 4 3}$ millions de dollars US à la formation des employés, avec des programmes adaptés à tous les niveaux de qualification.

- L'éducation des enfants des employés : 141 écoles installées sur les plantations, 1149 enseignants qui assurent l'éducation de 23370 enfants.

- En partenariat avec plusieurs universités indonésiennes, la compagnie a apporté son soutien à $\mathbf{5 2 8}$ étudiants depuis 2007 afin de leur permettre de poursuivre des études supérieures.

- En matière de santé, les employés et leur famille peuvent profiter gratuitement des structures créées par la compagnie, qui comprennent actuellement 121 dispensaires employant $\mathbf{2 3 0}$ docteurs et infirmières. Près de 840 patients y ont recours chaque jour.

- Par ailleurs, la compagnie prend à sa charge une grande partie des frais médicaux des employés et de leur famille, lors de consultations ou interventions extérieures.

- Les salaires des employés et bénéfices divers sont largement supérieurs aux standards minimum du pays.

- En matière d'environnement, outre le plan de la compagnie de compléter la certification RSPO de l'ensemble de ces plantations avant la fin de 2015, la compagnie a mis en œuvre des programmes opérationnels et/ou de recherche dans les domaines aussi variés que la prévention de pollution par les engrais, l'utilisation préférentielle de la gestion intégrée des maladies, avec recours minimal aux pesticides, l'utilisation de sources d'énergie renouvelable pour ses usines, la recherche d'évaluation des niveaux d'émission de gaz à effet de serre et leur réduction, aussi bien lors des changements d'utilisation des terrains (pas de développement sur forêt primaire, pas de développement sur tourbes) que lors des pratiques culturales dans les plantations établies.

Près de $\mathbf{2 3} \mathbf{5 0 0}$ hectares de terre ont été mis en conservation, du fait de leur valeur de biodiversité ou de leur fonction écologique spécifique.

La compagnie a débuté sa route vers la durabilité dès les débuts de son développement. Alors que les aspects économiques et sociaux ont constitués les premières mesures destinées à assurer la durabilité de la production, les actions plus tournées vers l'environnement se sont développées progressivement, au fur et à mesure des connaissances dans ce domaine relatif aux problèmes liés aux ressources naturelles et aux risques d'impacts environnementaux, ainsi qu'à l'existence de solutions opérationnelles permettant de les corriger et de les prévenir. La figure 3 résume ces principales initiatives et mesures prisent par PT. Smart au cours des 4 dernières décennies.

\section{Les années 1980 : les débuts} de la compagnie et les premieres mesures économiques et sociales

Les années 1980 ont vu la mise en place des premiers programmes de développement, de pratiques de culture permettant d'arriver au potentiel de production pour les zones pédoclimatiques concer- nées par les implantations de la compagnie, et l'adoption des principes de gestion intégrée des ravageurs (Integrated Pest Management) permettant de protéger les cultures avec un recours minimal aux pesticides. Un département de R\&D est alors créé, afin de mettre en place un premier système de gestion rationnel de la nutrition minérale.

Côté social, le programme de la compagnie s'oriente surtout vers les mesures en faveur des employés pour assurer une stabilité et une efficience du personnel, en fournissant des conditions de travail attractives en termes de salaires, d'accès à l'éducation des enfants et aux services de santé.

\section{Les années 1990 : \\ un développement intense}

Un programme systématique de développement de formation des employés de la compagnie et de construction d'écoles sur les plantations pour la scolarisation de leurs enfants se mit en place, accompagné par un programme similaire de développement de structure de soins médicaux (encadré 1). Par ailleurs, des actions d'aides aux communautés locales (entretien d'infrastructures, etc.) se développent (Corporate Social Responsability).

\section{La gestion des déchets : compatibilité entre mesures environnementales et agronomiques}

Les années 1990 ont vu la mise en place d'un programme intensif relatif aux aspects environnementaux liés aux sous-produits résultant de l'extraction de I'huile : alerté par le risque environnemental potentiel des déchets liquides (entre 2,7 et 2,8 tonnes d'effluent par tonne $d^{\prime}$ 'huile produite) et solides ( 1 tonne de rafles par tonne d'huile produite), et prenant avantage de la valeur nutritionnelle de ces sous-produits et de la non-présence de métaux lourds, la compagnie décida de valoriser ces déchets sous forme d'engrais organiques, épandus dans les parcelles généralement sous forme fraîche ou après co-compostage tel cette usine située à 

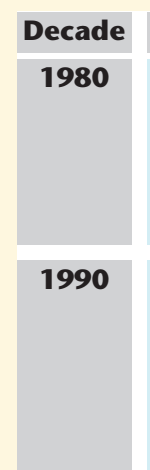

2000

Economy Aspects
Plantation development
Field practices
IPM
R\&D

Plantation development

Plantation consolidation

IPM

R\&D +

Fertiliser Management

\section{Plantation development Plantation consolidation Field practices: SOPs IPM \\ R\&D ++ \\ Fertiliser Management Breeding \\ Molecular biology}

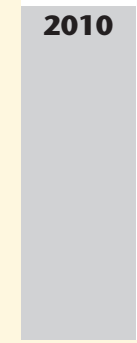

Plantation development
Plantation consolidation
Field practices: SOPs
IPM
R\&D +++
Fertiliser Management
Breeding
Molecular biology
Field practices: SOPs

Social Aspects
Company labour:
Wages $\left(^{*}\right)$
Children school (pupils)
Health
Community
CSR

Company labour:
Wages
Children school
Health
Training $\left({ }^{* *}\right)$
Community
CSR +

Company labour:
Wages
Children school
Health
Training
Education (Students)
Community
CSR ++
Soclalization-FPIC
R\&D

Company labour:
Wages
Children school
Health
Training
Education $\left({ }^{* *}\right)$
Community
CSR +++
FPIC
R\&D

$\quad$ Environment Aspects
IPM

IPM
By-Products management
Zero burning policy
Impact assessment
R\&D

IPM
By-Products management
Zero burning policy
Impact assessment
RSPO
ICOPE (sharing information)
Biodiversity (HCVF)
R\&D +

Figure 3. PT. Smart : les principales initiatives prises au cours des 3 dernières décennies.

Jambi (figure 4). Un programme de recherche fut mis en place et les résultats permirent d'intégrer ces engrais organiques aux plans de fertilisation et de maintien de la fertilité du sol. Les autres déchets solides (coques et fibres) étaient déjà utilisés pour assurer une énergie renouvelable pour le fonctionnement des usines.

La gestion de la nutrition minérale de l'ensemble des plantations de la compagnie relève d'un dispositif fondé sur I'association entre un programme expérimental comprenant un réseau d'essais de fertilisation de référence, et un suivi étroit de l'état de nutrition des arbres par analyse de tissu végétal (diagnostic foliaire annuel). Ce travail est effectué en collaboration avec le Cirad. Les pratiques de culture sont revues périodiquement afin de tenir compte des avancées technologiques et les connaissances agronomiques.

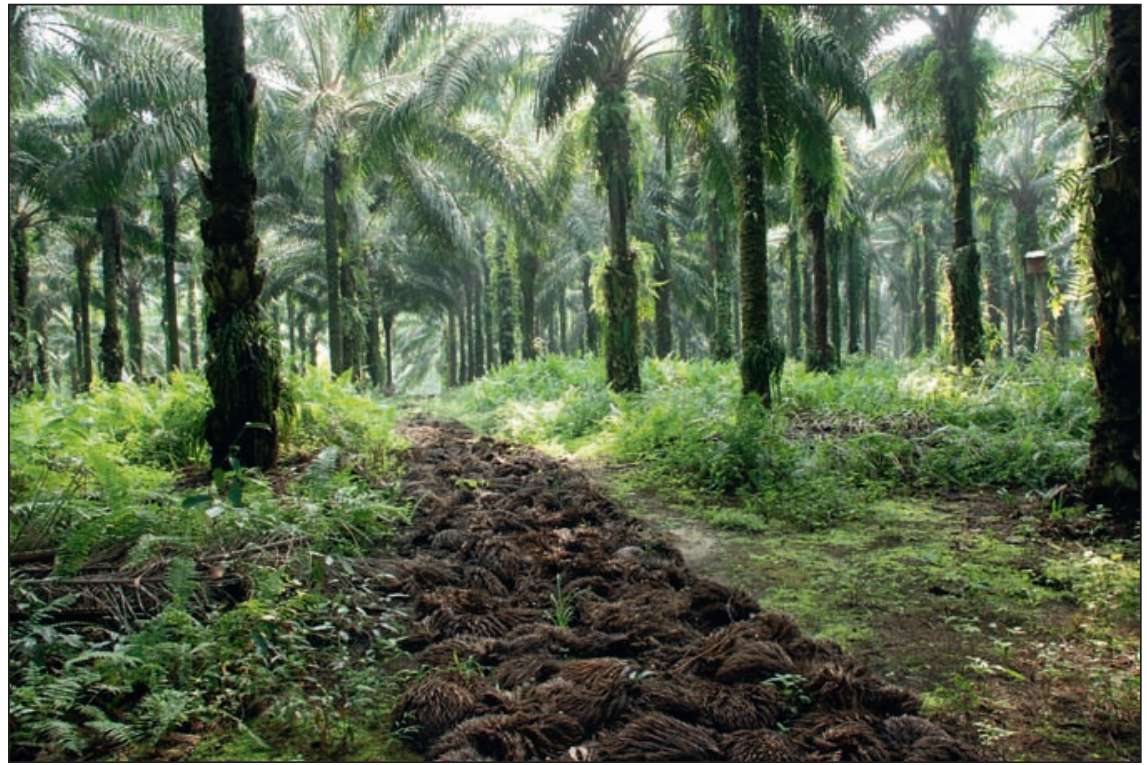

Figure 4. Recyclage de matieres organiques en plantation. 
Suite aux sévères conditions climatiques enregistrées en 1997, avec le phénomène El Nino, résultant en de gigantesques incendies et une couverture de fumée sur l'ensemble de la région, la compagnie enregistra de fortes chutes de teneurs en huile dans les fruits, liées au faible rayonnement solaire (Caliman et Southworth, 1998). PT. Smart pris aussi conscience des possibilités de développement des nouvelles plantations par les moyens mécaniques et adopta le zero-burning dès 1998. Cette mesure permettait en outre de conserver la matière organique du précédent cultural, facteur important pour maintenir la fertilité physique, chimique et biologique des sols.

Les années 2000 : des changements accélérés

La crise économique observée en Asie du Sud-Est à la fin des années 1990 met en évidence l'importance de préparer des améliorations de performance et de maîtrise des coûts, tout en considérant les connaissances grandissantes sur les impacts environnementaux. Cette situation conduisit PT. Smart à accélérer la mise en place d'un programme d'amélioration végétale à moyen et long terme par voie conventionnelle, appuyé par des travaux en biologie moléculaire, et à plus court terme par le développement de technique de multiplication végétative des meilleurs palmiers. Parallèlement, un programme d'amélioration de la diversité génétique du palmier fut initié, en partenariat avec plusieurs compagnies indonésiennes et en collaboration avec plusieurs pays tropicaux. Par ailleurs une meilleure intégration des recherches dans les différentes disciplines, agronomie, amélioration des plantes, protection des cultures, est mis en place, avec des programmes transversaux.

\section{Les petits planteurs}

(smallholders) : une part

importante et intégrale

de la compagnie

Participant depuis le milieu des années 1990 au développement de programmes de plantations de palmiers à huile pour les petits planteurs, généralement organisés par le gouvernement avec un cahier des charges précis concernant aussi bien la mise en place que I'encadrement durant la période d'exploitation, PT. Smart décide d'intensifier son rôle auprès de ces petits planteurs afin de leur assurer des revenus plus conséquents.

Ainsi, depuis le milieu des années 2000, PT. Smart utilise sur les programmes villageois que la compagnie développe la même source de semences à haut potentiel de production que pour ses propres plantations. Auparavant, ces programmes étaient développés à partir de semences extérieures dont la qualité, bien que probable, ne pouvait pas être garantie par la compagnie puis qu'elle n'intervenait pas dans le processus de production. L'équipe assurant la supervision de ces programmes est également renforcée sur chaque unité afin d'apporter un soutien technique plus intense. Plus récemment, des blocks de démonstration incluant une optimisation de la gestion de la fertilisation sont mis en place. Par ce programme, la compagnie met les petits planteurs dans une position très favorable pour leur assurer des performances supérieures, pour leur bénéfice, mais aussi pour le bénéfice de la compagnie et du pays tout entier.

Sur chaque site, les planteurs peuvent choisir le degré de partenariat avec la société : d'un contrat de simple vente de fruits à une gestion totalement intégrée à celle des plantations de la compagnie. Dans chaque cas, un service de vulgarisation et de recommandations est fourni au planteur. De même, la compagnie a intégré l'ensemble de ces plantations dans son programme de certification RSPO, les premiers audits externes devant avoir lieu en 2011. Ils se poursuivront ensuite afin d'être complétés sur l'ensemble des unités au plus tard en 2015, comme l'ensemble des unités propres de la compagnie.

\section{Adoption de techniques prouvées scientifiquement, assurées économiquement, et opérationnelles}

De manière générale, la stratégie de la compagnie est d'adopter toute mesure et pratique agricole ayant été prouvée, et de faire des recherches et tests concernant celles à confirmer. Dans ce dernier cas, l'approche consiste souvent à s'associer avec d'autres partenaires de l'industrie du palmier à huile et/ou d'institutions de recherche. Ainsi, par exemple concernant la réduction des gaz à effet de serre, la stratégie de la compagnie pour réduire son empreinte de carbone consiste (GAR, 2011) :

- d'une part, à mettre en pratique les techniques ayant été prouvées telles le recyclage des coques et fibres comme matière première pour la fourniture d'énergie aux usines d'extraction de I'huile; au recyclage en engrais organique des déchets solides (rafles) et liquides (effluents); à l'adoption du zero-burning lors de la préparation des terrains ; aux efforts d'augmentation de la teneur en carbone organique des sols ; à la conservation des sols tourbeux non encore développés, etc. ;

- d'autre part, de travailler avec les institutions et/ou organismes de recherche afin de définir les méthodes de mesure et d'évaluation ; par exemple, participation au groupe de travail sur les gaz à effet de serre de RSPO ; aux études concernant l'évaluation d'une méthode de calcul de l'empreinte carbonée de plantations en diverses situations, avec I'ICRAF (Van Noordwijk et al., 2010), etc.

\section{La formation et l'éducation : pilier des performances actuelles et futures}

Des programmes de sponsorship pour les enfants des employés montrant une aptitude aux études supérieures dans les domaines de l'agriculture et de l'ingéniérie sont organisés afin de faciliter I'accès aux universités locales. Les futurs diplômés auront des débouchés dans l'entreprise, et l'entreprise a ainsi accès à une source de talents. Le développement continu des structures de formation des travailleurs et des cadres, aussi bien anciens que nouvellement recrutés, permet à la compagnie de poursuivre l'amélioration de ses performances par la formation de son personnel (encadré 1).

\section{Un service de santé pour tous}

Dans le domaine de la santé, le recrutement généralisé sur chaque unité, ou groupe d'unités, de médecins permet un accès facilité aux soins dans des conditions toujours améliorées.

\section{L'émergence des connaissances relatives aux problèmes environnementaux}

Les années 2000 correspondent à l'émergence des connaissances relatives 
aux problèmes environnementaux, liés à la confirmation du phénomène du réchauffement climatique, mais également relatifs à la gestion des ressources naturelles et au risque de pollution. Parallèlement, une prise en compte différente des intérêts des populations locales par rapport aux intérêts des populations globales des pays concernés est recommandée par les ONG sociales. Cette dernière approche s'est traduite par une socialisation de plus en plus poussée des nouveaux projets de développement et l'introduction du concept de "Free Prior Informed Conscent " exigeant une action d'information et d'analyse des projets, de leur impact attendu et possible, devant permettre aux populations locales d'effectuer des choix renseignés.

\section{RSPO : une participation \\ de PT. Smart active, constructive, mais toujours vigilante}

L'environnement prend une importance particulière, suite à l'intensification des connaissances, à une communication plus globale et à des phénomènes climatiques plus extrêmes.

La prise en compte de l'ensemble des piliers identifiés pour le développement durable (économie, social et environnement), conduit l'industrie du palmier à huile à la création de RSPO en 2003 (formalisée en 2004), sous l'initiative de plusieurs acteurs de la filière. PT. Smart y participe depuis la première table ronde en 2003 et adhère formellement à l'initiative en 2005.

La création de RSPO peut être considérée comme un tournant pour l'industrie de I'huile de palme. Elle fut la solution nécessaire pour répondre aux critiques grandissantes contre la profession, qui a la particularité d'exercer dans des domaines très sensibles : zone tropicale, pressions sur la forêt tropicale, menaces sur la biodiversité, émissions de gaz à effets de serre liées principalement aux changements d'utilisation des terres (déforestation), populations locales aux moyens limités, le tout se traduisant bien trop souvent par des jugements extérieurs faisant plus appel aux sentiments émotionnels des populations et consommateurs occidentaux qu'à la rationalité scientifique.

La participation de PT. Smart à RSPO se veut active et constructive, mais toujours vigilante afin d'assurer l'opérationnalité des mesures prises et recommandées aux planteurs. Plusieurs représentants de la compagnie participent et contribuent aux divers groupes de travail de RSPO à différents niveaux, jusqu'à la présidence de certains d'entre eux (par exemple, celle du groupe de travail concernant l'interprétation nationale des principes et critères de durabilité pour I'Indonésie). La compagnie participera également à diverses initiatives, par exemple le groupe de travail en Indonésie concernant les mécanismes de compensation de pertes éventuelles de biodiversité, etc., et bien d'autres groupes de travail, institutionnels ou informels.

\section{ICOPE : une conférence internationale pour partager expériences et solutions}

Conscient de l'importance grandissante des problèmes environnementaux à prendre en compte par l'industrie, réalisant le manque de connaissances dans nombre de domaines, voulant promouvoir les échanges et expériences, prenant également un tournant dans son rôle de leader pour l'industrie du palmier à huile, PT. Smart décide en 2006 d'organiser la première conférence internationale sur le palmier à huile et l'environnement, avec comme objectif : "to highlight environmental issues, sharing experience on possible solutions and resources, not only for the benefit of the environment, but also for the industry itself ". Pour cela, la compagnie demande à WWF, ONG environnementale particulièrement active dans la recherche de solutions, et au Cirad, centre de recherche reconnu pour sa capacité $d^{\prime}$ approche scientifique dans I'analyse des phénomènes et de conduite de programmes de recherches scientifiques et finalisés, de s'associer à ce projet. Fort du succès de la première conférence organisée en 2007, I'expérience est institutionnalisée avec un événement ICOPE organisé tous les 2 ans (www.icope-series.com).

\section{HCV : un concept important, mais des difficultés initiales en ressources humaines pour I'appliquer}

L'un des points traités lors de la première conférence ICOPE de novembre 2007 concerna les zones à haute valeur de biodiversité (HCV), dont le concept, introduit par RSPO en 2005, posera des problèmes à la compagnie entre 2007 et 2009, certainement en grande partie à l'origine des attaques organisées contre la compagnie par certaines ONG environnementales en 2009 et 2010. En effet, la rareté des équipes indépendantes formées à I'identification des zones HCV, I'absence d'outils opérationnels adaptés au cas du palmier à huile pour sa mise en œuvre, les difficultés institutionnelles pour conserver des zones non développées au sein de concessions allouées par les autorités au développement de projets agricoles, conduiront la compagnie à effectuer des choix des concessions à analyser en fonction risques environnementaux préalablement identifiés par les études d'impact traditionnelles. Malgré cela, plus de 23000 ha de concession ont été conservées (figures 5 et 6) pour leur valeur environnementale particulière (biodiversité, fonction spécifique, etc.) (encadré 2).

\section{0-2011 : des engagements renouvelés}

2010-2011 fut une période riche en événements et décisions, avec la confirmation d'une approche holistique du développement durable dans ces 3 piliers fondamentaux.

\section{La réponse aux ONG : dialogue, transparence et recherches de solutions}

La fin des années 2000 fut donc celle des attaques appuyées de certaines ONG, considérant certainement que PT. Smart, devait donner l'exemple du développement durable à l'ensemble de l'industrie du palmier à huile.

Parmi les allégations émises contre la compagnie, trois principales concernaient des enjeux environnementaux :

- la destruction de zones de haute valeur de conservation forestière (HCVF) ;

- d'une manière générale, la destruction de " rainforest " ou forêt tropicales qui, pour les personnes occidentales, équivaut généralement aux forêt primaires ; - la plantation sur tourbes.

La réponse de PT. Smart sera fondée sur trois points :

1) Réaffirmer ses engagements relatifs à l'environnement, en particulier la conservation des zônes de forêt primaire, de tourbes et d'espaces à haute valeur de conservation (biodiversité). 


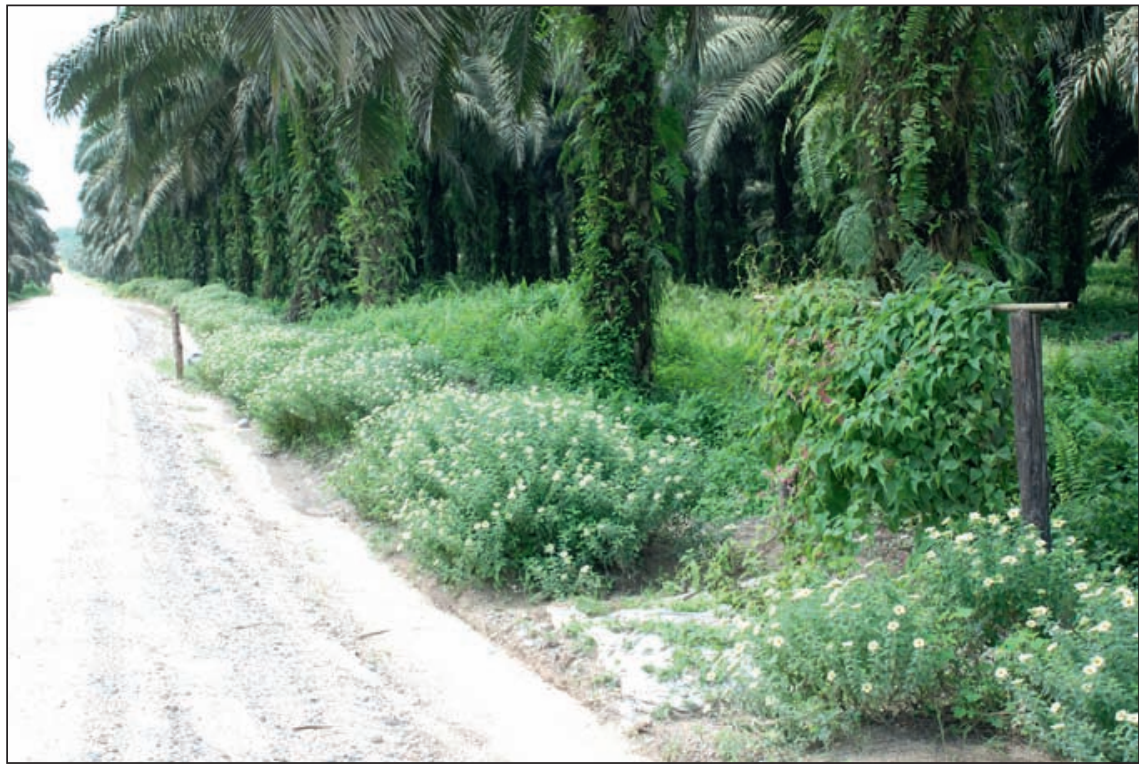

Figure 5. Plantes bénéfiques le long des chemins.

2) Faire vérifier par des organismes et experts indépendants l'existence, l'échelle et le cas échéant le contexte des erreurs commises par la compagnie citées dans les allégations des ONG.

3) Revisiter les procédures techniques internes et de gouvernance de la compagnie afin d'éviter à l'avenir toute erreur possible en les faisant certifier.

L'ensemble sera fait dans une transparence totale (lettre du 4 février 2010; rapports IVEX disponibles sur Www. smart-tbk.com), en accord avec sa stratégie de dialogue telle qu'appliquée

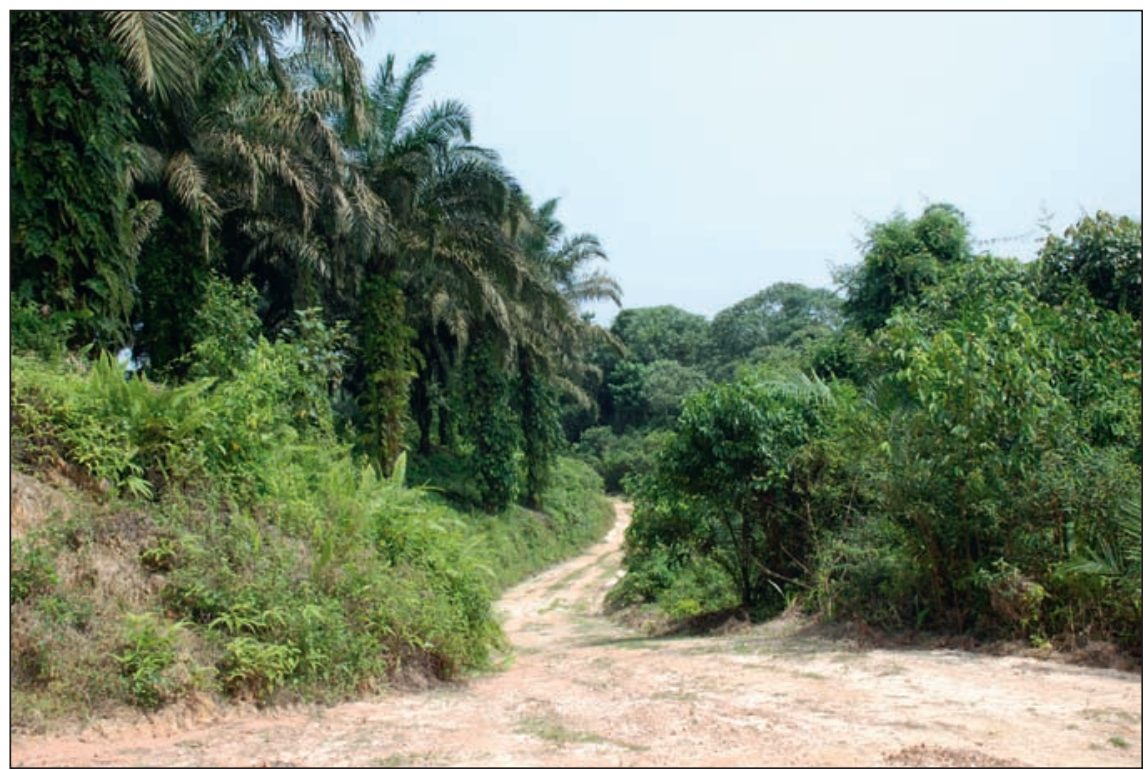

Figure 6. Zone de bas-fond conservée.
RSPO une procédure de "grievence" existait. Ceci conduit dans les mois qui suivirent à un sentiment de doute de la part de nombre de planteurs concernant $\mathrm{RSPO}$ et sa capacité de résoudre ce type de conflit.

Ce n'est que neuf mois après le début du "conflit", bien après la remise du rapport par PT. Smart au public, que RSPO pris I'initiative de mettre en route la procédure de grievance prévue par les statuts de l'organisme, et demanda à PT. Smart de prendre les mesures nécessaires à la correction des situations mentionnées dans le rapport.

Une nouvelle étape se mit en place en 2011, avec un nouveau partenariat établi avec The Forest Trust, organisme choisi pour sa connaissance de l'approche environnementale dans les processus de production, des pratiques prenant plus encore en compte les demandes des consommateurs pour une production responsable. événements, et en admettant que la compagnie ne prétendait nullement au zéro-défaut mais qu'elle se considérait comme étant une compagnie responsable dans le sens anglo-saxon du terme.

Singulièrement, RSPO fut relativement absente dans un premier temps du processus, alors que les allégations relevaient de critères émis par RSPO, alors que les premières sanctions furent émisent par un membre fondateur de RSPO (Unilever) envers un autre membre de RSPO (PT. Smart), et qu'au sein de

\section{Conclusion}

Les accusations contre la société PT. Smart relatives à la non-durabilité de son système de production ont été l'occasion pour la compagnie :

1) de confirmer ses engagements en faveur de la production d'huile de palme durable ;

2) d'affirmer et d'affiner son approche, en reconnaissant certaines erreurs, en les corrigeant et en mettant en place un système de prévention ;

3) de renforcer son rôle de leadership dans l'industrie pour le bénéfice de tous, c'est-à-dire de la compagnie elle-même, de l'industrie du palmier à huile et de I'Indonésie.

L'amélioration permanente (continuous improvement) étant l'un des critères de valeur interne de la compagnie (sharevalue de la compagnie), c'est dans ce cadre et avec cet esprit que la compagnie s'efforce de se développer en mettant en place depuis sa fondation des techniques de production et de gestion les mieux à même d'assurer une production durable avec les connaissances du moment. L'un des principaux défis est d'appréhender quelles sont les informations disponibles qui relèvent purement de la connaissance, et celles qui peuvent être traduites en pratiques opérationnelles. Ce challenge devenant plus aigu au cours de la dernière 


\section{Encadré 2 \\ What are HCVFs?}

High Conservation Value Forests are defined as the forest areas required to maintain or enhance the High Conservation Values (HCVs) that have been identified. There are six categories of HCVs (see below), covering both ecological and social values. A full HCVF assessment would need to cover all six values. Sometimes it has proved useful to undertake partial assessments focusing, for example, on just the ecological or social values. However, these cases are not considered full HCVF assessments.

The six types of High Conservation Value

- HCV1: Globally, regionally or nationally significant concentrations of biodiversity values.

For example, the presence of several globally threatened bird species within a Kenyan montane forest.

- HCV2: Globally, regionally or nationally significant large landscape-level forests.

For example, a large tract of Mesoamerican lowland rainforest with healthy populations of jaguars, tapirs, harpy eagles and caiman as well as most smaller species.

- HCV3: Forest areas that are in or contain rare, threatened or endangered ecosystems.

For example, patches of a regionally rare type of freshwater swamp forest in an Australian coastal district.

- HCV4: Forest areas that provide basic services of nature in critical situations (e.g. watershed protection, erosion control).

For example, forest on steep slopes with avalanche risk above a town in the European Alps.

- HCV5: Forest areas fundamental to meeting the basic needs of local communities.

For example, key hunting or foraging areas for comminities living at subsistence level in a Cambodian lowland forest mosaic.

- HCV6: Forest areas critical to local communities' traditional cultural identity. For example, sacred burial grounds within a forest management area in Canada. Source: High conservation value forest: the concept in theory and practice. WWF International, 2007. 25 p décennie où le temps entre acquisition des connaissances et exigence de mise en pratique se rétréci.

L'amélioration de la durabilité n'est donc pas, dans le cas de PT. Smart, une nouvelle approche ou un nouveau concept, mais une route continue sur laquelle il est vrai la compagnie progresse à vitesse variable selon les connaissances, les outils opérationnels disponibles et le cadre institutionnel local.

\section{RÉFÉRENCES}

Caliman JP, Southworth A. Effect of drought and haze on the performance of oil palm. In: International Oil Palm Conference 23-25 September 1998, Bali, Indonesia.

FAO. State of the Worlds Forest, 2011. ISBN 978-92-5r-r106750-5.

GAR. Annual Report 2009. 2010. www. goldenagri.com.sg.

GAR. Annual Report 2010. 2011. www. goldenagri.com.sg.

IPOC. Indonesian Palm Soil Statistics 2009. Indonesian Palm Oil Commission. Ministry of Agriculture. 2010. ISSN 1907-7548

Oil World (2010).

RSPO. RSPO Principles and Criteria for Sustainable Palm Oil Production; Including Indicators and Guidance. October 2007. www. rspo.org.

Van Noordwijk M, Dewi S, Khasanah N, et al. Estimating carbon footprint from biodiesel production in oil palm: methodology and results from two sites in Indonesia. In: International Conference on Oil Palm and the Environment ICOPE 2010. 23-25 February 2010. Bali. Indonesia. 